\title{
Spatially Resolved Investigation of the Optical and Structural Properties of CuCl Thin Films on Si
}

\author{
Barry Foy ${ }^{a^{*}}$, Enda McGlynn ${ }^{\mathrm{a}}$, Aidan Cowley ${ }^{\mathrm{b}}$, Patrick J. McNally ${ }^{\mathrm{b}}$ and Martin O. \\ Henry $^{\mathrm{a}}$
}

\author{
${ }^{a}$ School of Physical Sciences, National Centre for Plasma Science and Technology, Dublin City University, \\ Glasnevin, Dublin 9, Ireland \\ ${ }^{b}$ Nanomaterials Processing Laboratory, Research Institute for Networks and Communications Engineering \\ (RINCE), School of Electronic Engineering, Dublin City University, Dublin 9, Ireland
}

\begin{abstract}
CuCl}$ thin films grown on (100) $\mathrm{Si}$ by thermal evaporation are studied by means of low temperature photoluminescence (PL) and reflectance spectroscopies. Spatially and wavelength resolved room temperature cathodoluminescence $(\mathrm{CL})$ imaging of the surface of the $\mathrm{CuCl}$ samples in a scanning electron microscope (SEM) has also been performed. The reflectance spectra are modeled using a dielectric response function with various models involving dead layers and reflected waves in the thin film and the exciton-polariton structure obtained is compared to other studies of $\mathrm{CuCl}$. The modeling is shown to match the experimental data quite well when a dead layer is included at the air/CuCl and $\mathrm{CuCl} / \mathrm{Si}$ interfaces. Some inconsistencies between the CL spectra and those measured by PL and reflectance have been observed. The effects of changing the accelerating voltage of the probe from $10 \mathrm{keV}$ to the range $1-5 \mathrm{keV}$ to allow depth analysis of the CL are reported, in order to pinpoint the spatial origin of the CL emission within the thin film.
\end{abstract}

Keywords: $\mathrm{CuCl}$, cathodoluminescence, photoluminescence, exciton, polariton, reflectance.

PACS: 78.20.-e, 78.40.-q, 78.55.-m, 78.60.-b, 78.66.-w, 73.20.Mf, 73.61.Le, 78.20.Bh, 78.40.Fy, 78.60.Hk,

*Author to whom correspondence should be addressed: barry.foy2@mail.dcu.ie

\section{INTRODUCTION}

Wide band gap materials have been studied extensively for a range of applications such as UV light emitting diodes, diode lasers and detectors [1]. Most of these efforts have focused on II-VI and III-nitride material systems, with the latter being the most productive for applications to date. A fundamental problem with these systems is the large lattice mismatch $\left(\sim 13 \%\right.$ [2]) between the $\mathrm{GaN}$ epitaxial layers and suitable substrates (e.g. $\mathrm{SiC}, \alpha-\mathrm{Al}_{2} \mathrm{O}_{3}$ ), which results in high densities of threading dislocations. With a band gap of $3.39 \mathrm{eV}$, a large exciton binding energy of $190 \mathrm{meV}$, and a lattice mismatch with cubic $\mathrm{Si}$ of $<0.4 \%$ at room temperature [3], $\mathrm{CuCl}$ is not hindered in this regard. $\mathrm{CuCl}$ is closely lattice-matched to both $\mathrm{Si}$ and $\mathrm{GaAs}$ and is an ideal candidate for the development of hybrid electronic-optoelectronic platforms. The low lattice mismatch should allow for low defect density $\mathrm{CuCl}$ growth on silicon substrates. The optical properties of $\mathrm{CuCl}$ thin films, their detailed understanding and optimization, are key to potential uses of this material in optoelectronic devices.

The purpose of this paper is to report low temperature reflectance and PL data from thin $\mathrm{CuCl}$ films on (100) $\mathrm{Si}$ substrates, to model the exciton-polariton structure underlying such phenomena, and to compare and contrast these data with room temperature CL images and spectra of the same samples in order to develop a more detailed understanding of the optical properties of such films. 


\section{EXPERIMENTAL TECHNIQUES}

$\mathrm{CuCl}$ thin films are grown on Si substrates with (100) surface orientation approximately $2 \mathrm{~cm}$ by $1.5 \mathrm{~cm}$ in size cut from a 4 inch silicon wafer. These wafers are single-sided, polished, p-type boron-doped silicon with a resistivity in the range of $0-20 \Omega \mathrm{cm}$. Prior to deposition, the substrates were degreased using Decon solution and organic solvents. The substrate native oxide is removed using dilute HF. Commercially available $\mathrm{CuCl}$ beads with $99.99 \%$ purity (Sigma-Aldrich) were evaporated from a quartz crucible onto the substrates using an Auto 306 Edwards evaporation system. The vacuum deposition was performed at $\sim 1 \times 10^{-6} \mathrm{mbar}$ at a rate of $\sim 0.5 \mathrm{nms}^{-1}$ until the desired thickness of $2000 \mathrm{~nm}$ was obtained. Due to the hygroscopic nature of $\mathrm{CuCl}$, it was essential that the samples were stored in a vacuum container at all times upon completion of the deposition. Exposure to atmospheric moisture in air causes the optical properties of the material to decay due to an increase in the level of hydrated oxyhalides of $\mathrm{Cu}^{++}$. [4]

The low temperature PL and reflectance scans were performed in a Janis CCS-500 closed-cycle cryostat in a He exchange gas environment. The temperature was controlled using an Oxford Instruments ITC-4 controller, resistive heater, and a $27 \Omega$ rhodium-iron resistance sensor on the sample holder. A deuterium lamp illuminated the samples during reflectance scans and a Kimmon IK Series He-Cd laser $325 \mathrm{~nm}$ was used for the PL scans. The angle of incidence for the reflectance scans was $<10^{\circ}$ to the c-axis. Spectra were acquired using a Bomem DA8 Fourier Transform spectrometer fitted with a Hamamatsu R1913 photomultiplier (PM) tube with the PM output matched to the spectrometer using a Bomem variable gain preamplifier. The resolution of the spectra acquired using this setup was $\sim 0.1 \mathrm{meV}$. CL scans were performed at room temperature using a Carl Zeiss EVO 50 Series SEM with an attached CL mirror and micrometer. Spectra were captured using the Gatan MonoCL monochromator with a 1200 lines/mm grating and a constant probe current of $300 \mathrm{pA}$.

\section{REFLECTANCE MODELLING}

The classical theory of exciton-polariton coupling given by Hopfield and Thomas [5] was used to model the reflectance data. Each of the experimental scans were performed at near normal incidence to minimize the influence of longitudinal exciton bands and allow our spectra to be modeled using a coupled two-exciton band model for the $\mathrm{CuCl} \mathrm{Z} \mathrm{Z}_{1,2}$ and $\mathrm{Z}_{3}$ exciton bands. These models combine Maxwell's boundary conditions with a form of ABCs. There are various possible $\mathrm{ABCs}$ reported but the 'Pekar $\mathrm{ABC}$ ' has proved adequate for modeling our spectra. This specifies that the total polarization due to each exciton branch disappears at the crystal interface or at a finite distance from the interface which gives rise to an exciton dead layer, as detailed elsewhere [6]. We have modeled each of these scenarios with our model 1 ignoring this dead layer and applying Pekar's ABC at the crystal interface and our model 2 including the dead layer thickness as a fitting parameter and applying Pekar's ABC at the dead layer boundary. Once Maxwell's equations are combined with Pekar's boundary conditions, we are left with a series of simultaneous equations for the boundary conditions. Details on the derivation of the models used is available in previous work [7].

To aid the fitting procedure, initial values are taken from the experimental data for the transverse and longitudinal frequencies of the excitons $Z_{1,2}$ and $Z_{3}$ which can be estimated from the maximum and minimum reflectivity at the expected spectral regions. The static background dielectric constant can be estimated from the average reflection coefficient far from the areas of interest. By varying parameters such as the $Z_{1,2}$ and $Z_{3}$ longitudinal and transverse exciton energies, the damping coefficient, exciton mass, and in the case of model 2 the dead layer thickness, the fitted reflectance spectra is optimized to the experimental data using a least squares procedure. For this we have used MATLAB [8].

For the first model we included the thickness of the material, the reflected waves from the $\mathrm{CuCl} / \mathrm{Si}$ interface, and a wave transmitted into the silicon substrate itself. The series of simultaneous equations obtained from the combination of Maxwell's equations with Pekar's boundary conditions are modified to include these parameters and re-expressed as a matrix equation. For the second model we included same parameters as the first, but with the exciton dead layers at the air/ $\mathrm{CuCl}$ and $\mathrm{CuCl} / \mathrm{Si}$ interfaces included. Each of these dead layers has its own thickness and the equations are modified and re-expressed as a matrix equation as before. 


\section{RESULTS AND DISCUSSION}

Figure 1 (a) shows the experimental reflectance data as well as the best fits for each of the two models used. We can see that both of the models quite closely match the experimental data with the dead layer model (model 2) being slightly closer to the measured experimental values for the $\mathrm{Z}_{3}$ exciton $(3.203 \mathrm{eV})$ and the $\mathrm{Z}_{1,2}$ exciton $(3.275 \mathrm{eV})$. This figure also displays the Fabry-Perot oscillations present throughout the spectra away from the exciton positions. The modeling allows us to ascertain the values for the thickness of the film, damping parameters of the excitons, and the effective masses all at the area under reflection. These values are found to be quite close to the literature values, which can be found elsewhere [9] [10] [11], indicating that our sample is of high quality with a low degree of stress or strain present [12]. There was a slight increase in the effective mass for the $Z_{3}$ exciton (2.5 instead of 2.4) and a larger thickness than deposited $(2.3 \mu \mathrm{m}$ instead of $\sim 2 \mu \mathrm{m})$. The difference in thickness could be due to a small variance throughout the sample due to the nature of the deposition.

We have also optically characterized the $\mathrm{CuCl}$ sample using photoluminescence. This is shown in figure 1 (b) with 3 main peaks visible. The free exciton peak $Z_{3}$ appears at $3.203 \mathrm{eV}$ which matches the value obtained from the reflectance data. The peak at $3.180 \mathrm{eV}$ is attributed to the bound exciton peak $\mathrm{I}_{1}$ which may be associated with an impurity, such as a $\mathrm{Cu}^{+}$vacancy [13]. The free bi-exciton PL band (M) is visible with the longitudinal (marked) at $3.165 \mathrm{eV}$ and the transverse at $3.173 \mathrm{eV}$, but of lower intensity. These are quite close to emissions previously recorded and with a similar order of intensity as well [14]. The broad PL band due to impurities, which can usually be observed on the low energy side of the $\mathrm{I}_{1}$ band, is not visible in this sample [15].
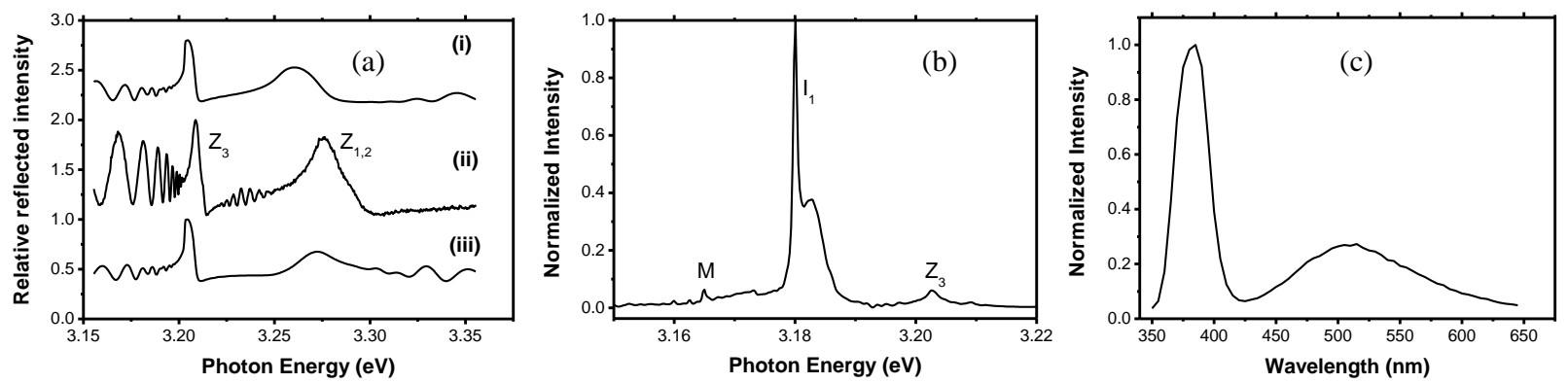

FIGURE 1. (a) Comparison of the reflectance spectra of the $\mathrm{CuCl}$ sample with computed reflectance spectra. (i) Model 1 using the thin film interference effects. (ii) Experimental data. (iii) Model 2 using the thin film interference effects combined with dead layers at both the $\mathrm{CuCl}$-Air and $\mathrm{CuCl}-\mathrm{Si}$ interfaces. The data is offset for clarity by 1 and 1.8 for the experimental data and the Model 1 fit respectively. The vertical scale is correct for each of the fits provided the appropriate offset is taken into account. Model parameters are given in table 1. (b) Photoluminescence spectra of the $\mathrm{CuCl}$ sample recorded at $20 \mathrm{~K}$ and normalized to the peak of maximum intensity. (c) Cathodoluminescence spectra of the $\mathrm{CuCl}$ sample recorded at room temperature with a beam energy of $4 \mathrm{keV}$ probe current of and normalized to the $390 \mathrm{~nm}$ peak.

Deep level defect emission at photon energies below the band edge region is a useful tool for judging material quality. An example of such emission is the $520 \mathrm{~nm}$ band in $\mathrm{CuCl}$ as seen in figure 1 (c). This was recorded at 4 $\mathrm{keV}$, as there was a clear increase in the level of intensity emitted from the sample at this point. Figure 2 shows the effect of the beam energy on the CL output of this $520 \mathrm{~nm}$ band, which allows us to judge the depth at which defect contributions become important in the thin film samples. Several different samples have been tested with similar outcomes. The sample of highest quality (in terms of integrated PL intensity detailed in previous work [16]) was chosen for examination.

Figure 2 (a) shows how the defect band at $520 \mathrm{~nm}$ increases relative to the $390 \mathrm{~nm}$ peak as the beam energy is increased. The small maximum in intensity between $1-2 \mathrm{keV}$ is due to the overall $\mathrm{CL}$ at these energies being of low intensity and may be ignored. As the beam energy was increased to $4 \mathrm{keV}$, there was a clear increase in the intensity emitted from the surface. Using a Monte Carlo simulation of the sample at this energy [17], the penetration depth was found to be over $140 \mathrm{~nm}$, which appears to be a crossover point within the material for a high level defect emission CL signal to be observed. Similarly between $20-30 \mathrm{keV}$ (and presumably beyond, although the limit of this SEM is $30 \mathrm{keV}$ ) we can see a leveling off of the relative $520 \mathrm{~nm}$ intensity. This can be attributed to the finite thickness of our sample. By tilting the sample in the SEM and imaging the edge of the material at a high resolution, the thickness of the $\mathrm{CuCl}$ layer was measured to be $\sim 2 \mu \mathrm{m}$. To ensure minimum variance in thickness, the $\mathrm{CL}$ spectra were recorded in close proximity to this edge region. A beam energy of $22 \mathrm{keV}$ or above will penetrate this layer and the Si substrate beneath. Previous studies [18] and our own observations have shown that the Si substrate does not contribute to the $520 \mathrm{~nm}$ or other emission bands. Therefore, the luminescence observed above this beam 

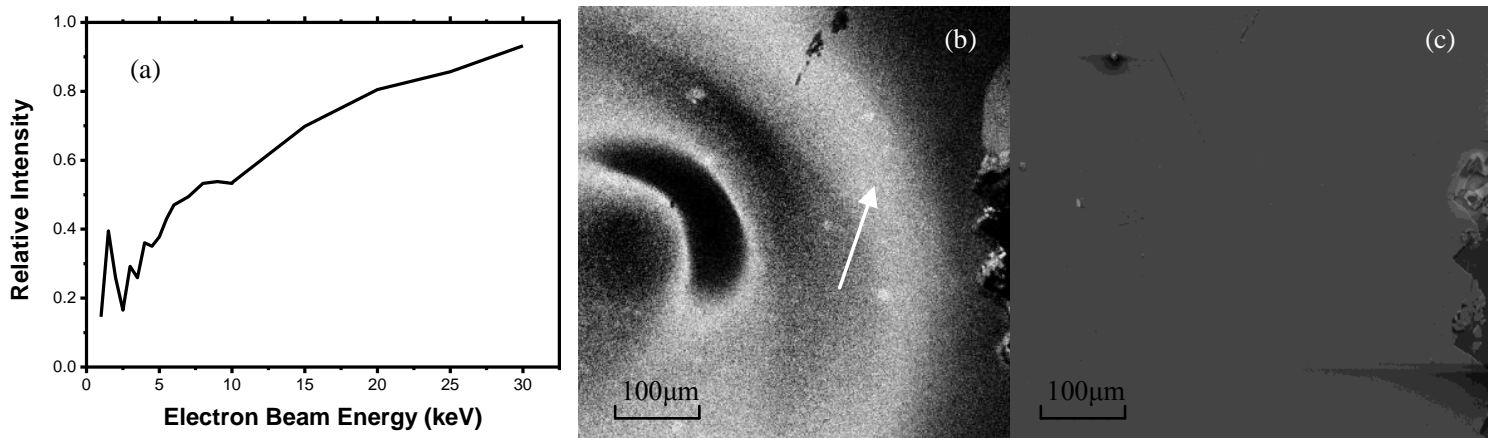

FIGURE 2. $\mathrm{CL}$ data from $\mathrm{CuCl}$ thin film sample. (a) Intensity of peak intensity of $520 \mathrm{~nm}$ emission band normalised to the band edge emittance peak as a function of beam energy. (b) The subtraction from each other of two CL images recorded in polychromatic mode at $4 \mathrm{keV}$ and $30 \mathrm{keV}$. Differences between the two beam energy images are clearly seen with the brighter material indicated by the arrow coming from the $30 \mathrm{keV}$ image. (c) Conventional (secondary electron) SEM image of the same area imaged by CL.

energy comprises luminescence from within the entire $\mathrm{CuCl}$ layer and no further changes in the band edge to deep level emission are seen, as expected. In figure 2 (b) and (c) we can see the increase in the beam energy has allowed us to image defects from beneath the surface of the material. The shapes in area indicated by the white arrow in (b) were not present in the $4 \mathrm{keV} \mathrm{CL}$ image or in the SEM image (c). Their position within the material can be ascertained by varying the beam energy and recording the initial and final positions of CL spectra change. Combined with the penetration depth of the beam energy this would allow their depth within the material to be ascertained.

\section{CONCLUSIONS}

$\mathrm{CuCl}$ thin films grown on $\mathrm{Si}(100)$ substrates have been optically characterized. Strong exciton and bi-exciton features have been observed at low temperature for both PL and reflectance spectra. The results of reflectance modeling using a dielectric response function have been shown to match our experimental spectra and can be used to characterize future samples. CL spectra at increasing levels of beam energy have been analyzed showing the increase in the defect band within $\mathrm{CuCl}$ relative to the $390 \mathrm{~nm}$ peak. Polychromatic $\mathrm{CL}$ imaging has been used to show the increase in detail obtained from this band beneath the sample's surface.

The authors gratefully acknowledge funding from Science Foundation Ireland under the Research Frontiers programme and Enterprise Ireland under the Proof of Concept programme.

\section{REFERENCES}

1. S. Nakamura, G.F., The Blue Laser Diode: GaN based Light Emitters and Lasers. Springer, Berlin, 1997.

2. Ambacher, O., Journal of Physics D: Applied Physics, 1998. 31(20): p. 2653.

3. Nishida, N., K. Saiki, and A. Koma, Surface Science, 1995. 324(2-3): p. 149-158.

4. Schwab, C. and A. Goltzene, Progress in Crystal Growth and Characterization of Materials, 1982. 5(3): p. $233-276$.

5. Hopfield, J.J. and D.G. Thomas, Physical Review, 1963. 132(2): p. 563-572.

6. Pekar, S.I., Soviet Physics-Solid State, 1962. 4(5): p. 953-960.

7. Fryar, J., et al., Nanotechnology, 2005. 16(11): p. 2625-2632.

8. http://www.mathworks.com/.

9. Soltani, M., et al., Computational Materials Science, 1995. 4(3): p. 263-268.

10. Sermage, B., M. Voos, and C. Schwab, Physical Review B, 1979. 20(8): p. 3245.

11. Ferhat, M., et al., Computational Materials Science, 2001. 20(2): p. 267-274.

12. Koda, T., et al., Physical Review B, 1972. 5(2): p. 705.

13. Nakayama, M., H. Ichida, and H. Nishimura, Journal of Physics-Condensed Matter, 1999. 11(39): p. $7653-7662$.

14. Shuh, D.K., et al., Physical Review B, 1991. 44(11): p. 5827.

15. Goto, T., T. Takahashi, and M. Ueta, Journal of the Physical Society of Japan, 1968. 24(2): p. 314.

16. Danieluk, D., et al., Journal of Materials Science-Materials in Electronics, 2009. 20: p. 76-80.

17. http://www.gel.usherbrooke.ca/casino/, CASINO (monte CArlo SImulation of electroN trajectory in sOlids).

18. Zamoryarskaya, M.V., V.I. Sokolov, and V. Plotnikov, Applied Surface Science, 2004. 234(1-4): p. $214-217$. 